\title{
Gut Microbiome Bridges Over Troubled Joints
}

\author{
Ji-Won Kim, M.D. ${ }^{1}$, Ji Hyeon Ju, M.D. ${ }^{2}$ \\ ${ }^{1}$ Division of Rheumatology, Department of Internal Medicine, Daegu Catholic University School of Medicine, Daegu, ${ }^{2}$ Division of \\ Rheumatology, Department of Internal Medicine, Seoul St. Mary's Hospital, College of Medicine, The Catholic University of Korea, Seoul, Korea
}

Osteoarthritis (OA) is the most prevalent joint condition causing chronic pain and disability. OA has generally been considered as a degenerative joint disease characterized by structural damage in the articular cartilage; however, $\mathrm{OA}$ is, in fact, more complex and heterogeneous involving cartilage, bone, and synovium further affected by multiple biomechanical and biochemical factors. With regard to these numerous factors, there is growing evidence that obesity and metabolic syndromes are associated with OA [1,2]. Reports of increased OA risk in non-weight bearing joints of obese patients suggest that metabolic factors can cause the perpetuation of OA $[3,4]$. Furthermore, it is speculated that adipokines and pro-inflammatory cytokines released from fat tissues might contribute to the development and progression of OA [5]. However, the exact mechanistic role of obesity on $\mathrm{OA}$ is yet to be fully understood.

Gut microbiome has gained much interest as a player in the pathogenesis of various diseases including OA. Physiologically, balanced community of microbial organisms resides in the gut and interacts with the human host by regulating the metabolism and immune responses of the host. However, disrupted gut microbial community could trigger dysregulated metabolic and immune responses. For instance, a study with gut microbiota from twin mice showed that "obese microbiota" transplantation transmitted obesity-associated metabolic phenotype, supporting the causal role of gut microbiota in obesity-related metabolic disorder [6]. Gut dysbiosis can also contribute to autoimmune and autoinflammatory diseases, such as inflammatory bowel disease, rheumatoid arthritis, spondyloarthritis, and systemic lupus er- ythematosus by increasing gut permeability, bacterial translocation, and immune system activation [7].

It is not clear whether gut microbiome has a direct causal relationship with OA or altered gut microbiome is a consequence of OA-related change. However, there are several preclinical studies which might support the direct connection between gut microbiome and OA. For instance, a study by Ulici et al. [8] observed less severe meniscus injury-induced $\mathrm{OA}$ in germ-free mice compared to specific pathogen free mice. Moreover, OA severity was enhanced in mice transplanted with fecal microbiota of OA patients with metabolic syndrome [9]. Another study has also seen reduced synovial inflammation and cartilage loss in obese OA mouse model after the modulation of gut microbiota by oligofructose (prebiotics) administration and the restoration of gut microbial composition (toward increased abundance of beneficial gut bacteria) [10]. These results suggest that gut microbiome might be linked to obesity and metabolic syndrome that could aggravate OA severity. In addition, gut microbiome could have a direct influence in cartilage, chondrocyte, and subchondral bone marrow damage [11]. Gut microbiome was recently found in human and mouse OA cartilage samples showing distinct composition and function compared to control cartilage samples, although this finding needs to be confirmed in further studies [12].

A recent study by Lee and Song [13] published in Journal of Rheumatic Diseases investigated a causal relationship between the gut microbiome and OA risk using a publicly accessible summary statistics datasets of gut microbiome, including the genome-wide association studies (GWAS) data of 3,326 individuals of European descent

\footnotetext{
Received : June 21, 2021, Accepted : June 21, 2021

Corresponding to : Ji Hyeon Ju (iD http://orcid.org/0000-0002-1381-5466

Division of Rheumatology, Department of Internal Medicine, Seoul St. Mary's Hospital, College of Medicine, The Catholic University of Korea, 222 Banpo-daero, Seocho-gu, Seoul 06591, Korea. E-mail : juji@catholic.ac.kr
}

Copyright (c) 2021 by The Korean College of Rheumatology.

This is an Open Access article, which permits unrestricted non-commerical use, distribution, and reproduction in any medium, provided the original work is properly cited. 
(as an exposure), summary GWAS data from 3,498 UK patients with knee and/or hip OA, and 11,009 controls of European descent (as an outcome). Because there is a lack of studies regarding the role of gut microbiome in human OA, this study has value in terms of examining the causal relationship between gut microbiome and OA using Mendelian Randomization design. Here, no causal relationship between gut microbiome and OA risk was found. However, we need to consider some crucial points while interpreting the results. First, there was no data regarding the outcomes of $\mathrm{OA}$, such as pain and inflammatory markers. A recent microbiome cohort study revealed that the abundance of certain gut microbe species was associated with more severe knee joint pain and inflammation in patients with OA [14]. Second, several metabolites and endotoxins released from gut microbes can also contribute to the pathogenesis of OA. For instance, lipopolysaccharide, a bacterial component playing as an endotoxin, was associated with OA severity and pain in patients with knee OA [15]. Third, the complexity of OA pathogenesis involving multiple factors (mechanic stress, metabolic, aging, hormonal, and genetic factors) makes it difficult to unravel the direct relationship between gut microbiome and OA [16].

In summary, gut microbiome may be involved in $\mathrm{OA}$ pathogenesis and affect $\mathrm{OA}$ outcomes by promoting local and systemic inflammation. This relationship may mean that "good" microbiome could reverse this pathogenic process. However, more data is needed to confirm this role of gut microbiome in the highly complex OA pathogenesis

\section{CONFLICT OF INTEREST}

No potential conflict of interest relevant to this article was reported.

\section{AUTHOR CONTRIBUTIONS}

Writing-original draft: J.W.K. Writing-review \& editing: J.W.K. and J.H.J.

\section{REFERENCES}

1. Yoshimura N, Muraki S, Oka H, Tanaka S, Kawaguchi H, Nakamura K, et al. Accumulation of metabolic risk factors such as overweight, hypertension, dyslipidaemia, and impaired glucose tolerance raises the risk of occurrence and progression of knee osteoarthritis: a 3-year follow-up of the ROAD study. Osteoarthritis Cartilage 2012;20:1217-26.

2. Monira Hussain S, Wang Y, Cicuttini FM, Simpson JA, Giles GG, Graves S, et al. Incidence of total knee and hip replacement for osteoarthritis in relation to the metabolic syndrome and its components: a prospective cohort study. Semin Arthritis Rheum 2014;43:429-36.

3. Dahaghin S, Bierma-Zeinstra SM, Koes BW, Hazes JM, Pols HA. Do metabolic factors add to the effect of overweight on hand osteoarthritis? The Rotterdam Study. Ann Rheum Dis 2007;66:916-20.

4. Yusuf E, Nelissen RG, Ioan-Facsinay A, Stojanovic-Susulic V, DeGroot J, van Osch G, et al. Association between weight or body mass index and hand osteoarthritis: a systematic review. Ann Rheum Dis 2010;69:761-5.

5. Visser AW, Ioan-Facsinay A, de Mutsert R, Widya RL, Loef M, de Roos A, et al. Adiposity and hand osteoarthritis: the Netherlands Epidemiology of Obesity study. Arthritis Res Ther 2014;16:R19.

6. Ridaura VK, Faith JJ, Rey FE, Cheng J, Duncan AE, Kau AL, et al. Gut microbiota from twins discordant for obesity modulate metabolism in mice. Science 2013;341:1241214.

7. Kim JW, Kwok SK, Choe JY, Park SH. Recent advances in our understanding of the link between the intestinal microbiota and systemic lupus erythematosus. Int J Mol Sci 2019;20:4871.

8. Ulici V, Kelley KL, Azcarate-Peril MA, Cleveland RJ, Sartor RB, Schwartz TA, et al. Osteoarthritis induced by destabilization of the medial meniscus is reduced in germ-free mice. Osteoarthritis Cartilage 2018;26:1098-109.

9. Huang Z, Chen J, Li B, Zeng B, Chou CH, Zheng X, et al. Faecal microbiota transplantation from metabolically compromised human donors accelerates osteoarthritis in mice. Ann Rheum Dis 2020;79:646-56.

10. Schott EM, Farnsworth CW, Grier A, Lillis JA, Soniwala S, Dadourian GH, et al. Targeting the gut microbiome to treat the osteoarthritis of obesity. JCI Insight 2018;3:e95997.

11. Berthelot JM, Sellam J, Maugars Y, Berenbaum F. Cartilage-gut-microbiome axis: a new paradigm for novel therapeutic opportunities in osteoarthritis. RMD Open 2019;5:e001037.

12. Dunn CM, Velasco C, Rivas A, Andrews M, Garman C, Jacob $\mathrm{PB}$, et al. Identification of cartilage microbial DNA signatures and associations with knee and hip osteoarthritis. Arthritis Rheumatol 2020;72:1111-22.

13. Lee YH, Song GG. The gut microbiome and osteoarthritis: a two-sample Mendelian randomization study. J Rheum Dis 2021;28:94-100.

14. Boer CG, Radjabzadeh D, Medina-Gomez C, Garmaeva S, Schiphof D, Arp P, et al. Intestinal microbiome composition and its relation to joint pain and inflammation. Nat Commun 2019;10:4881.

15. Huang ZY, Stabler T, Pei FX, Kraus VB. Both systemic and local lipopolysaccharide (LPS) burden are associated with knee OA severity and inflammation. Osteoarthritis Cartilage 2016;24:1769-75.

16. Bijlsma JW, Berenbaum F, Lafeber FP. Osteoarthritis: an update with relevance for clinical practice. Lancet 2011;377: 2115-26. 